\title{
Controlled oxidation of graphite to graphene oxide with novel oxidants in a bulk scale
}

\author{
Malgorzata Wojtoniszak • Ewa Mijowska
}

Received: 27 June 2012/ Accepted: 11 October 2012/Published online: 30 October 2012

(C) The Author(s) 2012. This article is published with open access at Springerlink.com

\begin{abstract}
In this study, a novel method of graphite chemical exfoliation to create graphene oxide (GO) is reported. Here, new oxidants were examined: a mixture of perchloric and nitric acids and potassium chromate. Furthermore, an effect of oxidation time, temperature of oxidation, and ultrasonication on graphite exfoliation degree was investigated. The obtained GOs were next reduced with glucose, used as a reducing agent. Detailed analysis of the materials indicated that when graphite was oxidized for $24 \mathrm{~h}$ at $50{ }^{\circ} \mathrm{C}$, 5-layered graphene was prepared. An effect of sonication process was also examined, and it was found to enhance the exfoliation to bilayer graphene. Furthermore, when time and temperature were increased to $48 \mathrm{~h}$ and $100{ }^{\circ} \mathrm{C}$, respectively, graphite was exfoliated to single-layer graphene. Therefore, it is believed that the proposed route can be applied for the preparation of graphene or few-layered graphene with defined number of layers upon the process parameters optimization and in a bulk scale. The materials were characterized with atomic force microscopy, Fourier-transform infrared spectroscopy, Raman spectroscopy, and X-ray diffraction.
\end{abstract}

M. Wojtoniszak $(\varangle) \cdot$ E. Mijowska

Institute of Chemical and Environment Engineering,

West Pomeranian University of Technology,

Pulaskiego 10, 70-322 Szczecin, Poland

e-mail: mwojtoniszak@zut.edu.pl
Keywords Graphite - Chemical exfoliation · Graphene oxide

\section{Introduction}

Graphene shows excellent properties, such as high intrinsic carrier mobility $\left(200,000 \mathrm{~cm}^{2} / \mathrm{V}^{3} \mathrm{~s}\right)$, superior thermal conductivity, and excellent mechanical strength and elasticity (Bolotin et al. 2008; Balandin et al. 2008; Lee et al. 2008). Since graphene was isolated in 2004 by Geim and coworkers (2004) using a scotch tape method, there have been many processes developed to produce graphene. Although the mechanical exfoliation of graphite leads to production of high-quality and high-mobility graphene flakes, this method is a time consuming process limited to a small scale production. One of the methods is thermal decomposition of $\mathrm{SiC}$ in temperature range between 1,000 and $1,500{ }^{\circ} \mathrm{C}$. Here, Si sublimate from the silicon carbide and leave behind a carbon-rich surface (Hass et al. 2008). This method requires controlling the number of graphene layers, repeatability of large area growths, and interface effects with the $\mathrm{SiC}$ substrate (Choi et al. 2010). The second method of graphene synthesis is chemical vapor deposition (CVD). This process is promising in large scale production of graphene. Many papers concerning CVD growth of graphene using different catalysts $(\mathrm{Ni}, \mathrm{Cu}, \mathrm{ZnS}, \mathrm{Fe}$ ) have been reported (An et al. 2011; Wei et al. 2009; Li et al. 2009; Reina et al. 2009; 
Obraztsov 2009). Graphene can be also synthesized via chemical exfoliation of graphite, where interlayer van der Waals forces are eliminated: chemical derivatization, intercalation, thermal expansion, the use of surfactants, and oxidation-reduction (Chakraborty et al. 2008; Lotya et al. 2009; Lee et al. 2008). The most common route to chemically exfoliate graphite is the use of strong oxidants to produce graphene oxide in a first stage. Graphite oxide was first prepared by B.C. Brodie, where it was treated with a mixture of potassium chlorate and nitric acid (Brodie 1859). Later, Hummers and Offeman (1958) used a mixture of sulfuric acid, sodium nitrate, and potassium permanganate to oxidize graphite. Recently, many papers reporting a modification of the Hummers method have been published. For instance, Marcano et al. (2010) used a mixture of sulfuric and orthophosphoric acids and potassium permanganate in the oxidation process, and found it improved an efficiency of the oxidation. This process is still widely investigated for two reasons: (1) it gives an opportunity to produce large scale graphene or few-layered graphene, (2) it has potential to provide the samples with controlled number of graphene layers upon the process parameters optimization.

In this study, novel oxidants of graphite, toward its chemical exfoliation, were examined: a mixture of perchloric and nitric acids and potassium chromate. Furthermore, an effect of oxidation time, temperature of oxidation and ultrasonication in exfoliation degree were investigated. The obtained materials were characterized with TEM, FT-IR, Raman spectroscopy, and XRD.

\section{Experimental}

\section{Materials}

Graphite was purchased from Alfa Aesar (synthetic, $99.9995 \%, 325$ mesh). Perchloric acid, nitric acid, hydrochloric acid, and ethanol were obtained from Chempur. $\mathrm{K}_{2} \mathrm{CrO}_{4}$ was bought from $\mathrm{POCH}$.

\section{Methods}

Here, three types of graphene oxides (GOs) were prepared. In the first procedure of GO1 synthesis, $1 \mathrm{~g}$ of graphite was dispersed in a mixture of perchloric and nitric acids (350 mL, 4:3-volume ratio), and next, $\mathrm{K}_{2} \mathrm{CrO}_{4}(6 \mathrm{~g})$ was added. The mixture was then heated to $50{ }^{\circ} \mathrm{C}$ and reaction was operated for $24 \mathrm{~h}$. The obtained mixture was next filtrated through polycarbonate (PC) membrane (Whatman $0.2 \mu \mathrm{m}$ ) and washed three times with ethanol $(200 \mathrm{~mL})$ and $10 \%$ hydrochloric acid $(200 \mathrm{~mL})$ to remove residual metal ions, and finally with distilled water until $\mathrm{pH}$ of the solution was 7 . Finally, the material was dried in air at $100{ }^{\circ} \mathrm{C}$ for $24 \mathrm{~h}$.

In the second method, prior heating a mixture of graphite, perchloric acid, nitric acid, and $\mathrm{K}_{2} \mathrm{CrO}_{4}$, an ultrasonication process was performed for $6 \mathrm{~h}$ at room temperature. After oxidation process, the purification, filtration, and drying steps were realized as in the first procedure, and finally GO2 was obtained.

In the third route, the time and the temperature of oxidation process were increased to $48 \mathrm{~h}$ and $100{ }^{\circ} \mathrm{C}$, respectively. The sonication, purification, filtration, and drying steps were carried out as in the second procedure to obtain GO3. After the purification process of the each graphene oxide, the content of impurities was determined with thermogravimetric analysis. It was estimated that $\mathrm{GO} 1, \mathrm{GO} 2$, and $\mathrm{GO} 3$ contain $0.11,0.19$, and $0.08 \mathrm{wt} \%$ of contaminants, respectively (data are not presented here).

Reduced graphene oxide (RGO) was synthesized with glucose using as a reducing agent (Zhu et al. 2010). In a typical procedure, graphene oxides (GO1, GO2, and GO3) were separately dispersed in $50 \mathrm{~mL}$ of water $(0.5 \mathrm{mg} / \mathrm{mL})$ and ultrasonicated for $2 \mathrm{~h}$. Next, $80 \mathrm{mg}$ of glucose was added to each homogeneous GO dispersion, and the mixtures were stirred for $30 \mathrm{~min}$. Then, $40 \mu \mathrm{L}$ of ammonia solution was added and reactions were heated to $95{ }^{\circ} \mathrm{C}$, stirring simultaneously for $2 \mathrm{~h}$. Next, the each reaction mixture was filtered through PC membrane $(0.2 \mu \mathrm{m}$ Whatman $)$. The obtained solid material was then washed with water and ethanol (3 times). Finally, the products (RGO1, RGO2, and RGO3) were dried in air at $100{ }^{\circ} \mathrm{C}$ for $24 \mathrm{~h}$.

The morphology of the obtained materials was characterized via atomic force microscopy (Nanoscope V MultiMode 8, Bruker). The measurements were done in air under ambient conditions. Raman measurements were performed on an In-Via Raman microscope (Renishaw) with excitation laser wavelengths of $785 \mathrm{~nm}$. Raman spectra were obtained from individual flakes deposited on $\mathrm{SiO}_{2} / \mathrm{Si}$ wafer $(300 \mathrm{~nm}$ $\mathrm{SiO}_{2}$ ) (Liana et al. 2010). The crystallographic 
structure of the samples were characterized by XRD analysis (X'Pert PRO Philips diffractometer) using a $\mathrm{CuK} \alpha$ radiation. FT-IR absorption spectra were recorded on Nicolet 6700 FT-IR Spectrometer.

\section{Results and discussion}

In this study, novel oxidants were used to create chemical exfoliation of graphite. In order to examine the efficiency of oxygen-containing functional groups introduction to carbon lattice, FTIR spectroscopy was used. Figure 1 presents FTIR spectra of GO1, GO2, and GO3. At each spectrum, several typical modes corresponded to oxygen-functional groups are detected. Here, stretching vibration modes of $\mathrm{C}-\mathrm{O}$ bonds arise at $1,099 \mathrm{~cm}^{-1}$. The peaks between 1,190 and $1,380 \mathrm{~cm}^{-1}$ are related with $\mathrm{C}-\mathrm{OH}$ stretching vibration (Xu et al. 2008). Deformation vibration modes of O-H groups are observed at $1,431 \mathrm{~cm}^{-1}$ (Stankovich et al. 2006). The bands appeared at $1,635 \mathrm{~cm}^{-1}$ correspond to adsorbed water molecules indicating hydrophilic properties of the material (Paredes et al. 2008). At approximately $1,709 \mathrm{~cm}^{-1} \mathrm{C}=\mathrm{O}$ stretching vibration of carboxyl groups are detected. The peaks at around $3,430 \mathrm{~cm}^{-1}$ arise from $\mathrm{O}-\mathrm{H}$ stretching vibration (Wojtoniszak et al. 2012). FTIR spectroscopy confirms successful oxidation. Furthermore, the intensities of the modes between 1,230 and $1,740 \mathrm{~cm}^{-1}$ increased in order of GO1, GO2, and $\mathrm{GO} 3$ which suggests an increase of the oxidation efficiency.

For further analysis XRD was used to monitor chemical exfoliation of graphite in a bulk scale. Figure 2 shows XRD patterns of graphite, GO1, GO2, and GO3. XRD pattern of graphite is dominated by single peak at $26.48^{\circ}$ corresponding to (002) plane (Liana et al. 2010). After oxidation process, the peak started to vanish, and new peaks arose at $24.28^{\circ}$, $24.78^{\circ}$, and $24.23^{\circ}$ in XRD patterns of GO1, GO2, and $\mathrm{GO} 3$, respectively. On the basis of Bragg's law, the interlayer spacing $\left(d_{001}\right)$ of $\mathrm{GO} 1, \mathrm{GO} 2$, and $\mathrm{GO} 3$ is 3.7, 3.6, and $3.7 \AA$, respectively. It is enhanced compared to that of graphite $(3.4 \AA$ ) (Fan et al. 2011). In GO2 and GO3 XRD patterns, more distinct decay of the peak at $26.48^{\circ}$ is observed. It suggests an enhanced exfoliation of graphite when oxidation was performed with ultrasonication treatment and when a time and a temperature was increased, also confirming the enhanced oxidation effect.

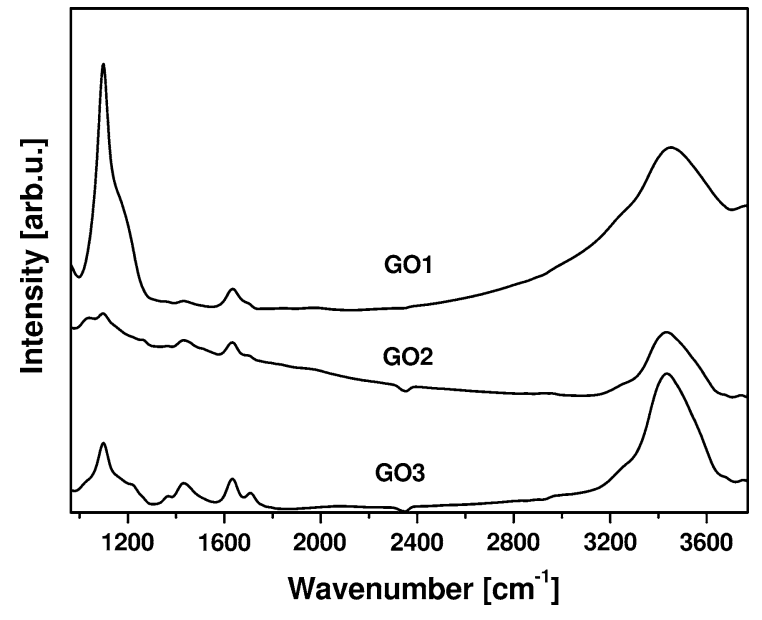

Fig. 1 FT-IR spectra of GO1, GO2, and GO3

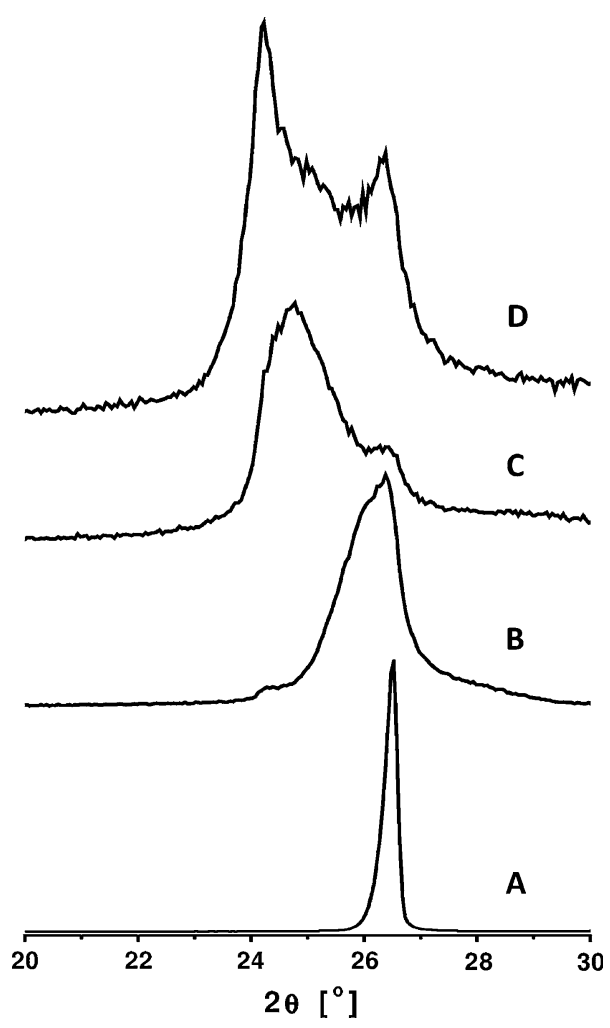

Fig. 2 XRD patterns of graphite (a), GO1 (b), GO2 (c), and $\mathrm{GO} 3(\mathbf{d})$

In order to determine the number of graphene layers, the samples underwent the reduction process. For reduced graphene oxide (RGO) preparation, glucose was used as a reducing agent. The obtained 
Fig. 3 Raman spectra of graphite (a), RGO1 (b), RGO2 (c), and RGO3 (d)

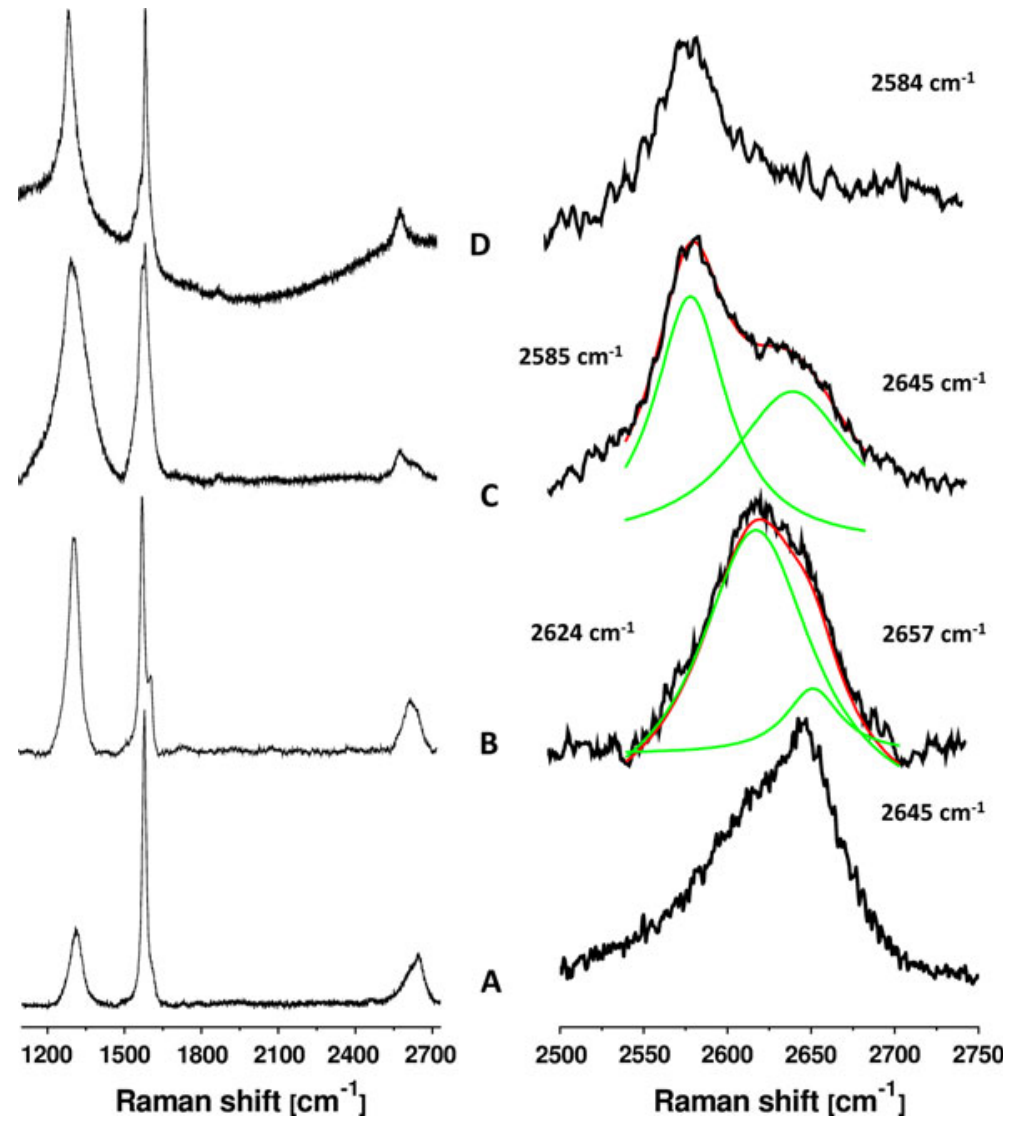

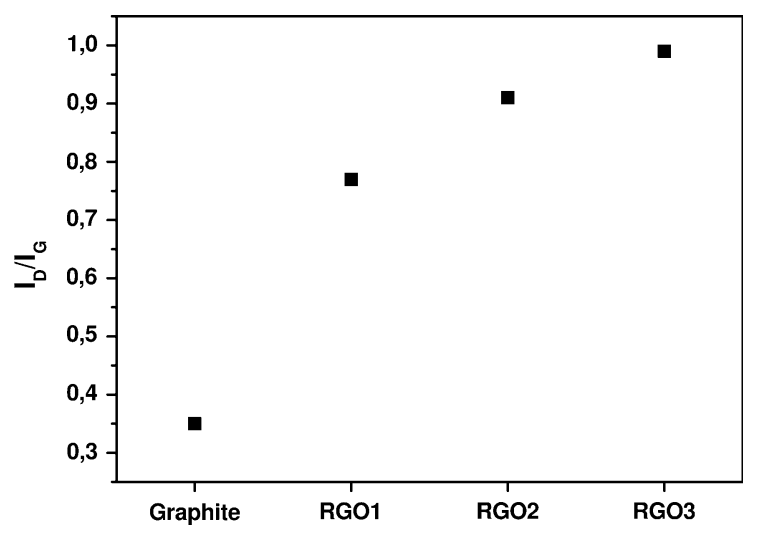

Fig. 4 Relative defects content in graphite, RGO1, RGO2, and RGO3 measured as ratio of $\mathrm{D}$ and $\mathrm{G}$ peaks intensities $\left(I_{\mathrm{D}} / I_{\mathrm{G}}\right)$

materials (RGO1, RGO2, and RGO3) were analyzed with Raman spectroscopy to characterize their structure, in terms of number of graphene layers and lattice defects. Figure 3 presents Raman spectra of graphite (A), RGO1 (B), RGO2 (C), and RGO3 (D). All spectra exhibit 3 typical modes related to graphitelike structure: a G band, a D band, and a 2D band
(Fig. 3, left panel). The G mode at approximately $1,580 \mathrm{~cm}^{-1}$ originates from the in-plane vibration of $\mathrm{sp}^{2}$ carbon atoms and is a doubly degenerate phonon mode ( $E_{2 \mathrm{~g}}$ symmetry) at the Brillouin zone center ( $\mathrm{Li}$ et al. 2007). The $\mathrm{D}$ band at roughly $1,320 \mathrm{~cm}^{-1}$ is a breathing mode of $A_{1 \mathrm{~g}}$ symmetry involving phonons near the $K$ zone boundary (Wilson et al. 2009). The 2D band arises from a two phonon double resonance Raman process (Ferrari and Robertson 2000). The 2D band is a key mode in identification of number of graphene layers. According to its position, shape and intensity, a thickness of graphene may be determined (Ferrari and Robertson 2000; Ni et al. 2008). Charlier et al. (2008) plotted the evolution of the 2D band as a function of layers in single-, bi-, and few-layer graphene. Briefly, a 2D band of bi- and few-layer graphene has 2 components $2 \mathrm{D}_{1}$ and $2 \mathrm{D}_{2}$. Increasing the number of layers leads to a significant increase of the relative intensity of the $2 \mathrm{D}_{2}$ peak and blue shift of the 2D peak. However, a 2D peak of single-layer graphene is composed of a single $2 \mathrm{D}$ peak, positioned at roughly $2,600 \mathrm{~cm}^{-1}$. Therefore, a Lorentzian 
Fig. 5 AFM images (left) and height profiles (right) of a 5-layered graphene of RGO1 (a), bilayer graphene of RGO2 (b), and singlelayer graphene of RGO3 (c). Scan size $500 \times 500 \mathrm{~nm}$
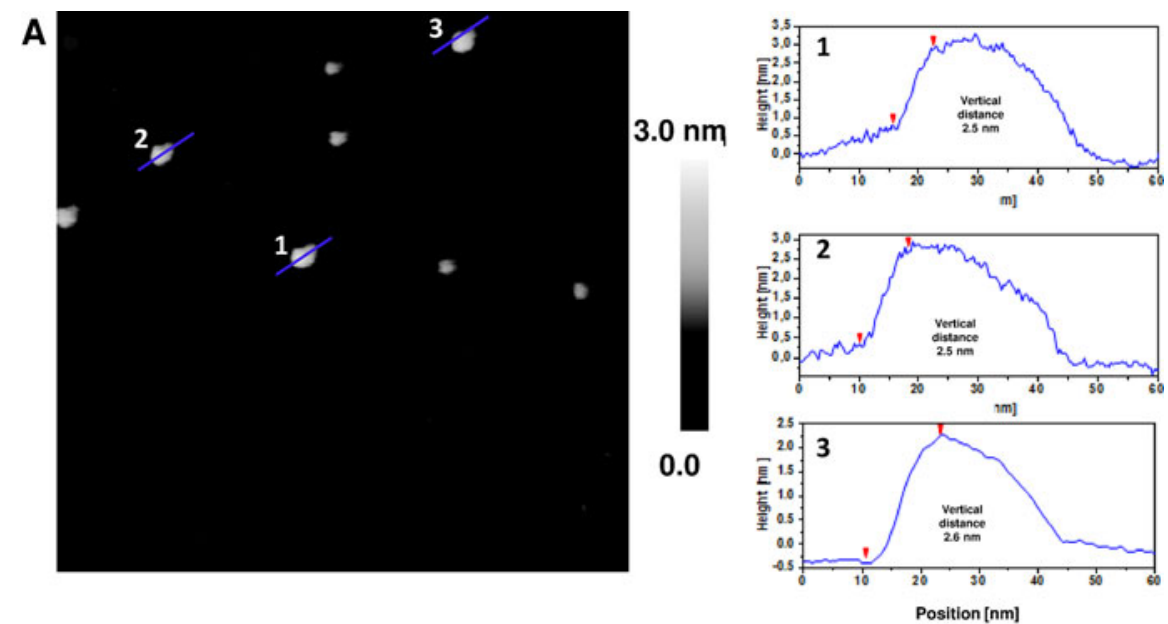

B
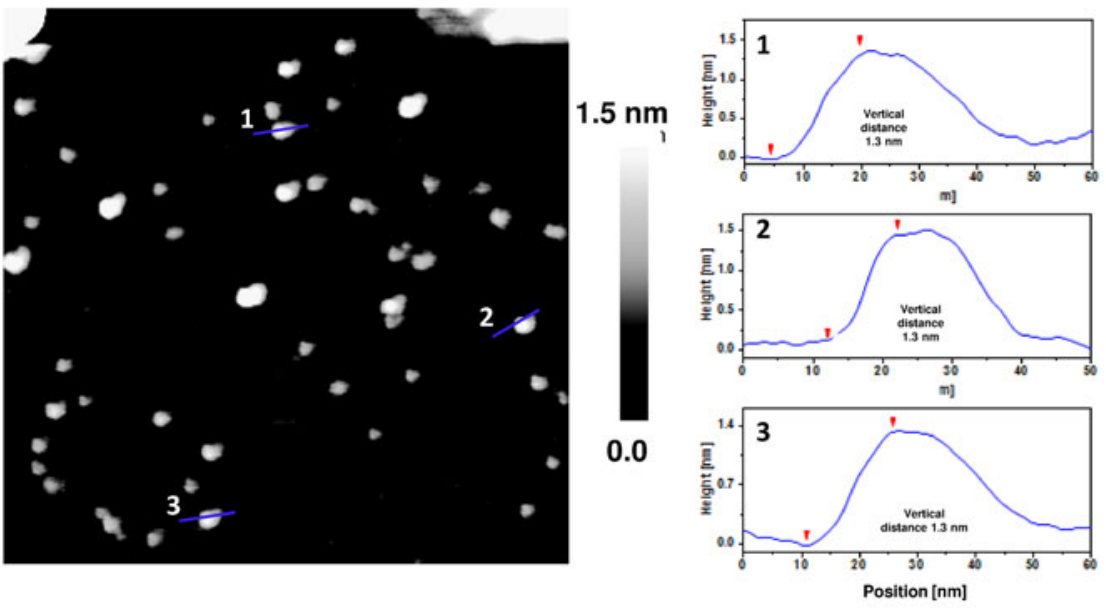

C

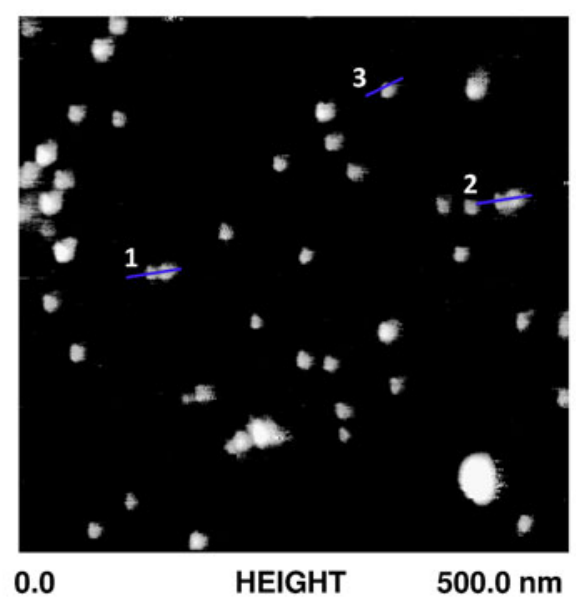

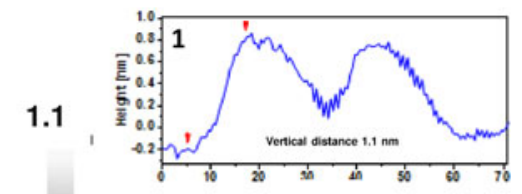
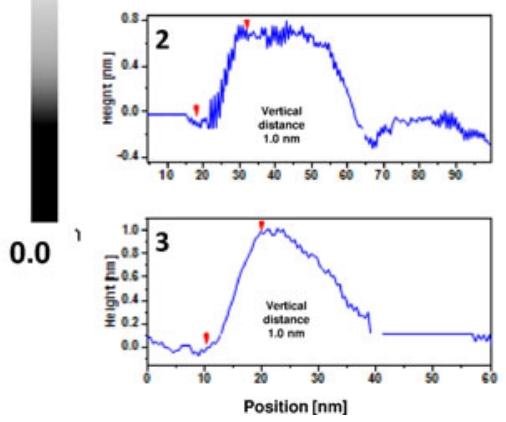

fitting of the RGOs spectra was performed and is presented in Fig. 3 (right panel). Here, 2D band of RGO1 differs from 2D band of graphite, and has two components: $2 \mathrm{D}_{1}$ and $2 \mathrm{D}_{2}$ with maximal intensity at 2,624 and $2,657 \mathrm{~cm}^{-1}$, respectively. It may be attributed to 5-layered graphene. The $2 \mathrm{D}_{2}$ peak of 
RGO2 decreases in comparison to RGO1. Furthermore, the 2D peak of RGO2 shifts to lower frequencies. These observations suggest the enhanced exfoliation of graphene when sonication was utilized leading to creation of bilayer graphene. It is clearly seen that $2 \mathrm{D}_{2}$ peak of RGO3 vanishes, and single $2 \mathrm{D}$ peak appears at $2,584 \mathrm{~cm}^{-1}$ attributed to single-layer graphene (Ferrari et al. 2006). It is known that a ratio between the intensities of the D and the $\mathrm{G}$ bands $\left(I_{\mathrm{D}} /\right.$ $I_{\mathrm{G}}$ ) determines relative defect content in the carbon lattice (Jorio et al. 2011). Therefore the $I_{\mathrm{D}} / I_{\mathrm{G}}$ ratios of RGOs spectra were calculated and results are presented in Fig. 4. It is shown that the $I_{\mathrm{D}} / I_{\mathrm{G}}$ ratio increases in order of RGO1, RGO2, and RGO3. This is related to the formation of vacancies and defects in carbon lattice, such as five- and seven-membered carbon rings, during the reduction process (Schniepp et al. 2006). This means that introduction of more oxygen-functional groups during the oxidation results in formation of more defects and vacancies in the final product-graphene.

To confirm the morphology of RGO1, RGO2, and RGO3, atomic force microscopy was applied. Figure 5 presents topography and height profiles of the prepared nanomaterials. The sizes of graphene flakes ranged from 30 to $70 \mathrm{~nm}$ in the each sample. The thickness of RGO1, RGO2, and RGO3 was 2.5-2.6, 1.3 , and $1.0-1.1 \mathrm{~nm}$, respectively. It was already reported that the thickness of a single-layer graphene on a substrate $\mathrm{SiO}_{2} / \mathrm{Si}$ with approximately $1 \mathrm{~nm} \mathrm{rms}$ roughness, would be $0.8-1.2 \mathrm{~nm}$. The enhanced thickness of monolayer graphene may be attributed to the interaction between the sample and the tip (Gupta et al. 2006; Nemes-Incze et al. 2008). In bi- or few-layered graphene, the thickness arises with additional layer and therefore, adding the expected $0.35 \mathrm{~nm}$ height corresponding to the native van der Waals inter-layer distance (Soldano et al. 2010). It was concluded that RGO1, RGO2, and RGO3 were composed of 5-layered, bilayer, and monolayer graphene, respectively (Li et al. 2008a, b). This observation is in full agreement with Raman response of the samples.

\section{Conclusions}

In summary, novel method of chemical exfoliation of graphite was presented. Here, novel oxidants were examined: a mixture of perchloric and nitric acids and potassium chromate, and an effect of oxidation time, temperature of oxidation, and ultrasonication in exfoliation degree were investigated. The presented methodology leads to creation of graphene with controlled number of layers: single-, bi-, and 5-layered graphene in a bulk scale.

Acknowledgments The authors are grateful for the financial support of National Science Center, nr 2011/01/N/ST5/02912.

Open Access This article is distributed under the terms of the Creative Commons Attribution License which permits any use, distribution, and reproduction in any medium, provided the original author(s) and the source are credited.

\section{References}

An H, Lee WJ, Jung J (2011) Graphene synthesis on Fe foil using thermal CVD. Curr Appl Phys 11:S81-S85. doi: 10.1016/j.cap.2011.03.077

Balandin AA, Ghosh S, Bao W, Calizo I, Teweldebrhan D, Miao F, Lau CN (2008) Superior thermal conductivity of singlelayer graphene. Nano Lett 8:902-907. doi:10.1021/ n10731872

Bolotin KI, Sikes KJ, Jiang Z, Klima M, Fudenberg G, Hone J, Kim P, Stormer HL (2008) Ultrahigh electron mobility in suspended graphene. Solid State Commun 146:351-355. doi:10.1016/j.ssc.2008.02.024

Brodie BC (1859) On the atomic weight of graphite. Proc R Soc Lond 10:249-259. doi:10.1098/rspl.1859.0007

Chakraborty S, Guo W, Hauge RH, Billups WE (2008) Reductive alkylation of fluorinated graphite. Chem Mater 20:3134-3136. doi:10.1021/cm800060q

Charlier JC, Eklund PC, Zhu J, Ferrari AC (2008) Electron and phonon properties of graphene: their relationship with carbon nanotubes. Top Appl Phys 111:673-709

Choi W, Lahiri I, Seelaboyina R, Kang YS (2010) Synthesis of graphene and its applications: a review. Crit Rev Solid State Mater Sci 35:52-71. doi:10.1080/1040843090350 5036

Fan ZJ, Kai W, Yan J, Wei T, Zhi LJ, Feng J (2011) Facile synthesis of graphene nanosheets via $\mathrm{Fe}$ reduction of exfoliated graphite oxide. ACS Nano 5:191-198. doi: $10.1021 / \mathrm{nn} 102339 \mathrm{t}$

Ferrari AC, Robertson J (2000) Interpretation of Raman spectra of disordered and amorphous carbon. Phys Rev B 61:14095-14107. doi:10.1103/PhysRevB.61.14095

Ferrari AC, Meyer JC, Scardaci V, Casiraghi C, Lazzeri M, Mauri F, Piscanec S, Jiang D, Novoselov KS, Roth S, Geim AK (2006) Raman spectrum of graphene and graphene layers. Phys Rev Lett 97:187401-187404. doi:10.1103/ PhysRevLett.97.187401

Gupta A, Chen G, Joshi P, Tadigadapa S (2006) Raman scattering from high-frequency phonons in supported $n$ graphene layer films. Nano Lett 6:2667-2673. doi:10.10 21/n1061420a 
Hass J, De Heer WA, Conrad EH (2008) The growth and morphology of epitaxial multilayer graphene. J Phys Condens Matter 20:323202-323229. doi:10.1088/0953-8984/20 132/323202

Hummers WS Jr, Offeman RE (1958) Preparation of graphitic oxide. J Am Chem Soc 80:1339. doi:10.1021/ja01539a017

Jorio A, Ferreira EHM, Cançado LG, Achete CA, Capaz RB (2011) Measuring disorder in graphene with Raman spectroscopy. Phys Status Solidi B 247:2980-2982. doi: 10.1002/pssb.201000247

Lee C, Wei X, Kysar JW, Hone J (2008) Measurement of the elastic properties and intrinsic strength of monolayer graphene. Science 321:385-388. doi:10.1126/science.115 7996

Li ZQ, Lu CJ, Xia ZP, Zhou Y, Luo Z (2007) X-ray diffraction patterns of graphite and turbostratic carbon. Carbon 45:1686-1695. doi:10.1016/j.carbon.2007.03.038

Li D, Mueller MB, Gilje S, Kaner RB, Wallace GG (2008a) Processable aqueous dispersions of graphene nanosheets. Nat Nanotechnol 3:101-105. doi:10.1038/nnano.2007.451

Li X, Wang X, Zhang L, Lee S, Dai H (2008b) Chemically derived, ultrasmooth graphene nanoribbon semiconductors. Science 319:1229-1232. doi:10.1126/science.115 2458

Li X, Cai W, An J, Kim S, Nah J, Yang D, Piner R, Velamakanni A, Jung I, Tutuc E, Banerjee SK, Colombo L, Ruoff RS (2009) Large-area synthesis of high-quality and uniform graphene films on copper foils. Science 324:1312-1314. doi:10.1126/science. 1171245

Liana P, Zhub X, Lianga S, Li Z, Yang W, Wang H (2010) Large reversible capacity of high quality graphene sheets as an anode material for lithium-ion batteries. Electrochim Acta 55:3909-3914. doi:10.1016/j.electacta.2010.02.025

Lotya M, Hernandez Y, King PJ, Smith RJ, Nicolosi V, Karlsson LS, Blighe FM, De S, Wang Z, McGovern IT, Duesberg GS, Coleman JN (2009) Liquid phase production of graphene by exfoliation of graphite in surfactant/water solutions. J Am Chem Soc 131:3611-3620. doi: 10.1021/ja807449u

Marcano DC, Kosynkin DV, Berlin JM, Sinitskii A, Sun Z, Slesarev A, Alemany LB, Lu W, Tour JM (2010) Improved synthesis of graphene oxide. ACS Nano 4:4806-4814. doi: $10.1021 / \mathrm{nn} 1006368$

Nemes-Incze P, Osváth Z, Kamarás K, Biró LP (2008) Anomalies in thickness measurements of graphene and few layer graphite crystals by tapping mode atomic force microscopy. Carbon 46:1435-1442. doi:10.1016/j.carbon.2008. 06.022
Ni Z, Wang Y, Yu T, Shen Z (2008) Raman spectroscopy and imaging of graphene. Nano Res 1:273-291. doi:10.1007/ s12274-008-8036-1

Novoselov KS, Geim K, Morozov SV (2004) Electric field in atomically thin carbon films. Science 306:666-669. doi: 10.1126/science. 1102896

Obraztsov AN (2009) Chemical vapour deposition: making graphene on a large scale. Nat Nanotechnol 4:212-213. doi:10.1038/nnano.2009.67

Paredes JI, Villar-Rodil S, Martínez-Alonso A, Tascón JMD (2008) Graphene oxide dispersions in organic solvents. Langmuir 24:10560-10564. doi:10.1021/la801744a

Reina A, Jia X, Ho J, Nezich D, Son H, Bulovic V, Dresselhaus MS, Kong J (2009) Large area, few-layer graphene films on arbitrary substrates by chemical vapor deposition. Nano Lett 9:30-35. doi:10.1021/n1801827v

Schniepp HC, Li JL, McAllister MJ, Sai H, Herrera-Alonso M, Adamson DH (2006) Functionalized single graphene sheets derived from splitting graphite oxide. J Phys Chem B 110:8535-8539. doi:10.1021/jp060936f

Soldano C, Mahmood A, Dujardin E (2010) Production, properties and potential of graphene. Carbon 48:2127-2150. doi:10.1016/j.carbon.2010.01.058

Stankovich S, Piner RD, Nguyen SBT, Ruoff RS (2006) Synthesis and exfoliation of isocyanate-treated graphene oxide nanoplatelets. Carbon 44:3342-3347. doi:10.1016/j. carbon.2006.06.004

Wei D, Liu Y, Zhang H, Huang L, Wu B, Chen J, Yu G (2009) Scalable synthesis of few-layer graphene ribbons with controlled morphologies by a template method and their applications in nanoelectromechanical switches. J Am Chem Soc 131:11147-11154. doi:10.1021/ja903092k

Wilson NR, Pandey PA, Beanland R, Young RJ, Kinloch IA, Gong L (2009) Graphene oxide: structural analysis and application as a highly transparent support for electron microscopy. ACS Nano 3:2547-2556. doi:10.1021/nn $900694 \mathrm{t}$

Wojtoniszak M, Zielinska B, Kalenczuk RJ, Mijowska E (2012) Photocatalytic performance of titania nanospheres deposited on graphene in coumarin oxidation reaction. Mater Sci Pol 30(1):32-38. doi:10.2478/s13536-012-0008-1

Xu Y, Bai H, Lu G, Li C, Shi G (2008) Flexible graphene films via the filtration of water-soluble noncovalent functionalized graphene sheets. J Am Chem Soc 130:5856-5857. doi: 10.1021/ja800745y

Zhu C, Guo S, Fang Y, Dong S (2010) Reducing sugar: new functional molecules for the green synthesis of graphene nanosheets. ACS Nano 4:2429-2437. doi:10.1021/nn1002387 